\title{
Targeted treatment with pazopanib in metastatic soft tissue sarcoma: Nearly complete response in two cases
}

\author{
ALI MURAT SEDEF ${ }^{1}$, FATIH KÖSE ${ }^{1}$, ÖZLEM DOĞAN ${ }^{2}$, TARKAN ERGÜN ${ }^{3}$, AHMET SEZER $^{1}$, \\ HÜSEYIN MERTSOYLU ${ }^{1}$, SADIK MUALLAOĞLU ${ }^{1}$, AYBERK BEŞEN ${ }^{1}$ and ÖZGUR ÖZYILKAN ${ }^{1}$ \\ Departments of ${ }^{1}$ Medical Oncology, ${ }^{2}$ Internal Medicine and ${ }^{3}$ Radiology, \\ Baskent University Adana Hospital, Yuregır, Adana, Turkey
}

Received June 25, 2014; Accepted October 22, 2014

DOI: $10.3892 / \operatorname{mco} .2014 .456$

\begin{abstract}
Soft tissue sarcomas (STS) are a group of rare mesenchymal cancers that include approximately 50 histological types and account for $1 \%$ of all adult cancers. The standard curative treatment option for localized disease is surgical resection and, if a surgically removed tumor exhibits high-risk characteristics, adjuvant chemotherapy and radiotherapy may be administered. Sarcoma presenting at an advanced stage has a dismal prognosis and survival has not markedly improved over the last 20 years. The standard first-line treatment for advanced STS, other than gastrointestinal stromal tumors, is cytotoxic chemotherapy. Therapies targeting pro-angiogenic factors have been a focus of drug development for STS over the last few years. Pazopanib, a multitargeted tyrosine kinase inhibitor, is a novel treatment option for patients with metastatic STS in the second-line setting. This is a presentation of 2 case reports of patients with metastatic STS who responded well to treatment with pazopanib.
\end{abstract}

\section{Introduction}

Soft tissue sarcomas (STS) are a heterogeneous group of rare tumors that arise from mesenchymal cells at various body sites $(1,2)$. The malignant precursor cell(s) may differentiate along one or several lineages, such as muscle, adipose, fibrous, cartilage, nerve or vascular tissue. STS has >50 distinct histological subtypes, with leiomyosarcoma, liposarcoma, synovial sarcoma, undifferentiated pleomorphic sarcoma and malignant peripheral nerve sheath tumors being among the most common (3). STS occurs accounts for $\sim 1 \%$ of malignancies in adults and $2 \%$ of the overall cancer mortality $(4,5)$. Approximately half of the patients diagnosed with STS

Correspondence to: Dr Ali Murat Sedef, Department of Medical Oncology, Baskent University Adana Hospital, Adana kisla saglik yerleskesi, Yureg1r, Adana 01130, Turkey

E-mail: alimuratsedef@gmail.com

Key words: response rate, pazopanib, metastatic soft tissue sarcomas, targeted therapy present with advanced/metastatic cancer and eventually succumb to their disease $(1,6)$. The median overall survival for advanced-stage disease was reported to range between 11 and 18 months $(7,8)$.

For advanced-stage STS, judicious use of cytotoxic therapy provides meaningful palliation and may prolong survival. The selection of systemic therapy may be individualized based upon several factors, including tumor histology, health status and preferences of the patients $(2,8,9)$. The European Society for Medical Oncology and the National Comprehensive Cancer Network treatment guidelines recommend anthracycline-based chemotherapy, primarily with doxorubicin, either as monotherapy or in combination with ifosfamide, as first-line treatment for the most advanced STS subtypes $(9,10)$. Pazopanib is a novel treatment modality and has been approved by the Food and Drug Administration and the European Medicines Agency as second-line treatment after a phase III trial of this drug reported a statistically significant increase in progression-free survival. Of note, only $6 \%$ of the patients exhibit a tumor response and in the majority of the cases, stable disease may be achieved. There remains the question of whether this drug may be used in highly symptomatic cases, as in some cases response rate may be a more important goal than survival benefit. In this study, we present 2 case reports of patients with STS who achieved a nearly complete radiological response to pazopanib.

\section{Case 1}

A 25-year-old female patient presented with a soft tissue mass at the level of right knee that had developed 3 years prior. The patient's past history was negative for systemic diseases and the laboratory evaluation was normal. We used positron emission tomography (PET)-computed tomography (CT) for initial staging and to exclude systemic metastases. The PET-CT showed a hypermetabolic lesion close to the right knee joint, filling the popliteal area, sized 110x82x160 mm (maximum standard uptake value, 10.7) (Fig. 1). The histopathological evaluation was compatible with a malignant mesenchymal tumor, namely a synovial sarcoma. The patient was treated with systemic neoadjuvant chemotherapy, followed by surgical resection in August, 2010. The patient also received adjuvant radiotherapy. 


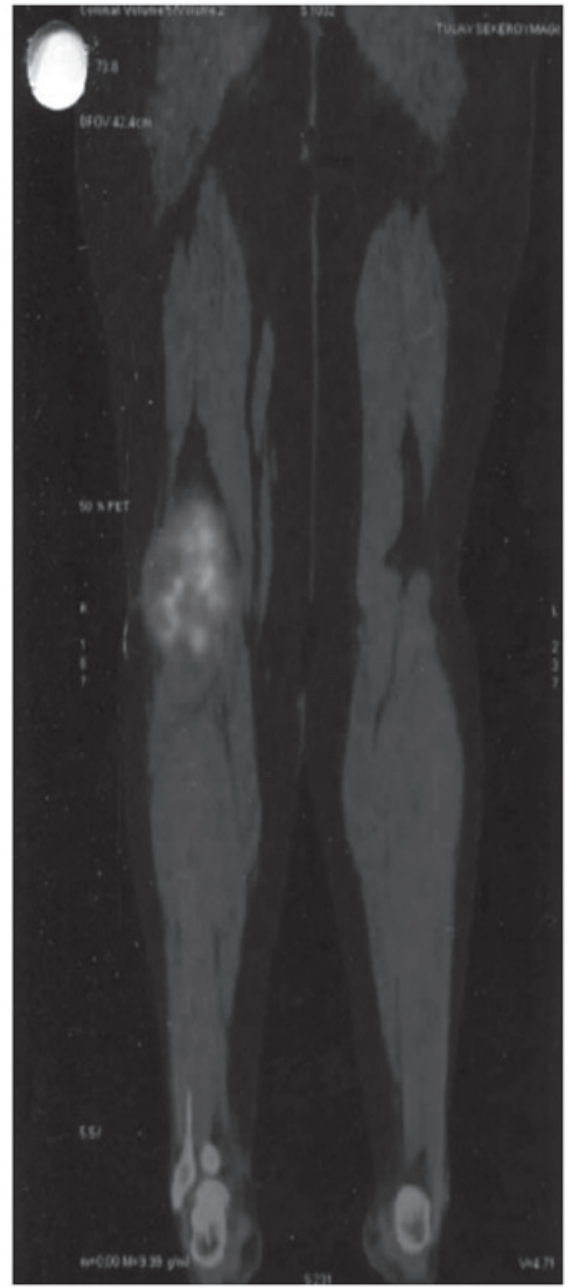

Figure 1. Case 1: Positron emission tomography-computed tomography showing a primary hypermetabolic lesion, sized 110x82x160 mm (maximum standard uptake value, 10.7), filling the entire popliteal fossa.

In September, 2012, the patient presented with dyspnea and her detailed evaluation with PET-CT revealed lung metastases, accompanied by right iliac chain and right inguinal lymphadenopathy (Fig. 2). The patient received treatment with a gemcitabine-docetaxel combination regimen, but the disease progressed. Subsequently, pazopanib ( $800 \mathrm{mg} / \mathrm{day})$ was initiated in January, 2013. The radiological evaluation in July, 2013 was compatible with nearly complete response (Figs. 3).

\section{Case 2}

A 50-year-old female patient presented with a right breast mass in July, 2010. The preoperative biopsy was compatible with the diagnosis of primary breast leiomyosarcoma and a right mastectomy was performed. The patient was treated with 6 courses of doxorubicin-based adjuvant chemotherapy. During surveillance, the patient presented with progressive dyspnea in September, 2012 and the PET-CT scan revealed lytic bone lesions and hypermetabolic metastatic lung nodules (Fig. 4). At the second-line setting, the patient was treated with docetaxel-gemcitabine and the progression-free survival with this regimen was 6 months; the maximal response, as evaluated by PET-CT, was stable disease. The patient received

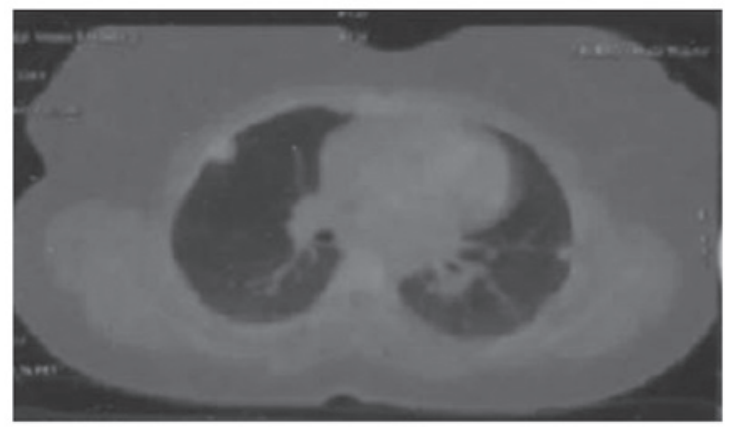

Figure 2. Case 1: Positron emission tomography-computed tomography revealed multiple hypermetabolic nodular lesions compatible with lung metastases.

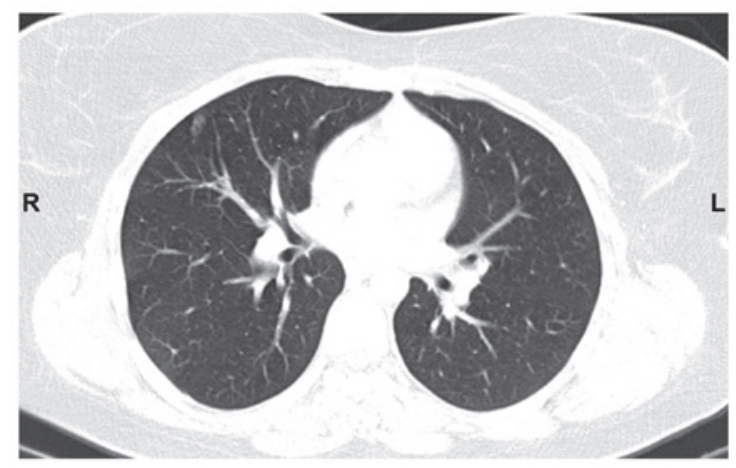

Figure 3. Case 1: Computed tomography evaluation following treatment with pazopanib revealed a good partial response.

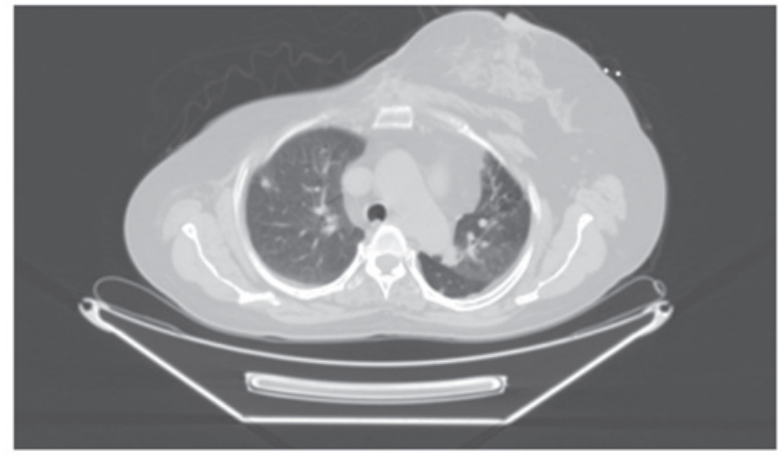

Figure 4. Case 1: Computed tomography evaluation following treatment with pazopanib revealed a good partial response.

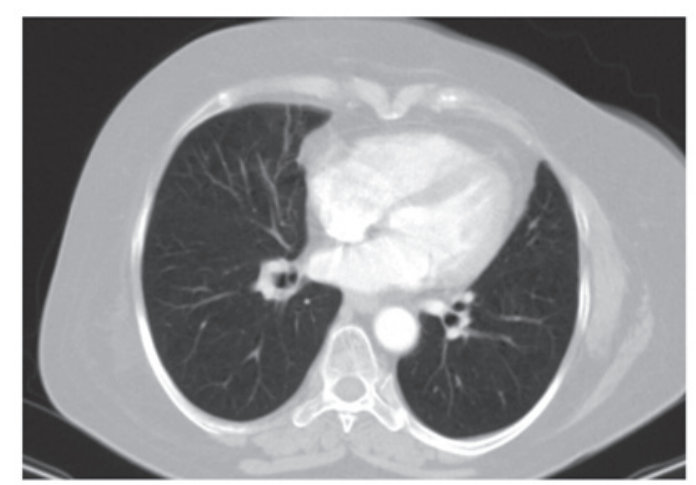

Figure 5. Case 2: Computed tomography evaluation demonstrating hypermetabolic osteolytic bone lesions and multiple metastatic lung nodules. 
palliative radiotherapy to the bone metastases in March, 2013. The radiological evaluation following radiotherapy showed progression of the lung metastases. Subsequently, pazopanib (800 mg/day) was initiated in May, 2013. The radiological evaluation in July, 2013 was compatible with nearly complete response (Fig. 5).

\section{Discussion}

Metastatic STS is an incurable disease and very little improvement regarding the treatment of advanced STS has been achieved over the last 2 decades, excluding imatinib treatment for gastrointestinal stromal tumors (GIST). Pazopanib, which is a synthetic indazolpyrimidine, is a multitargeted tyrosine kinase inhibitor, exhibiting activity against the vascular endothelial growth factor receptors 1,2 and 3, and the platelet-derived growth factor receptor (11). Single-agent pazopanib was found to be effective against various STS subtypes in a phase II clinical trial (12). Subsequently, a registration phase III study (PALETTE trial) was designed to compare pazopanib (800 mg daily) vs. placebo in 369 patients with a variety of histological subtypes, excluding liposarcomas or GIST, in the second-line setting (13). There was a statistically significant increase in PFS in the pazopanib group (4.6 vs. 1.6 months) and the survival benefit was consistent across all histological subtypes (14). However, although pazopanib significantly increased PFS in that study, the objective response rate was quite low and the best overall response was partial response in $6 \%$ of the pazopanib group. When evaluating pazopanib in the light of those trials, we may not recommend pazopanib for patients in need of fast symptomatic relief. However, when we used pazopanib in our first 2 cases as third-line treatment, a nearly complete response was achieved. On analyzing the demographic, clinical and histopathological characteristics of these 2 cases, including drug-related side effects, we were unable to identify a common point that may help explain why these 2 patients exhibited such a strong response to pazopanib.

In conclusion, we reported 2 cases that exhibited an unexpected, nearly complete response to anti-angiogenic treatment, with the aim to raise the question of whether we can define a specific group of patients who respond well to treatment with pazopanib.

\section{References}

1. Clark MA, Fisher C, Judson I and Thomas JM: Soft-tissue sarcomas in adults. N Engl J Med 353: 701-711, 2005.

2. Casali PG and Blay JY; ESMO/CONTICANET/EUROBONET Consensus Panel of Experts: Soft tissue sarcomas: ESMO Clinical Practice Guidelines for diagnosis, treatment and follow-up. Ann Oncol 21 (Suppl 5): v198-v203, 2010.

3. Cormier JN and Pollock RE: Soft tissue sarcomas. CA Cancer J Clin 54: 94-109, 2004.

4. Jain A, Sajeevan KV, Babu KG, Lakshmaiah KC: Chemotherapy in adult soft tissue sarcoma. Indian J Cancer 46: 274-287, 2009.

5. Weiss SW and Goldblum JR: General considerations. In: Enzinger and Weiss's Soft Tissue Tumors. Weiss SW and Goldblum JR (eds). 4th Edition. CV Mosby, St. Louis, MO, pp1-19, 2001.

6. Schöffski P: Pazopanib in the treatment of soft tissue sarcoma. Expert Rev Anticancer Ther 12: 711-723, 2012.

7. Italiano A, Mathoulin-Pelissier S, Cesne AL, Terrier P, Bonvalot S, Collin F, Michels JJ, Blay JY, Coindre JM and Bui B: Trends in survival for patients with metastatic soft-tissue sarcoma. Cancer 117: 1049-1054, 2011.

8. Grimer R, Judson I, Peake D and Seddon B: Guidelines for the management of soft tissue sarcomas. Sarcoma 2010: 506182, 2010.

9. Spira AI and Ettinger DS: The use of chemotherapy in soft-tissue sarcomas. Oncologist 7: 348-359, 2002.

10. NCCN: NCCN Clinical Practice Guidelines in Oncology: Soft Tissue Sarcoma, Volume V.1. 2011. National Comprehensive Cancer Network, 2011.

11. Schutz FA, Choueiri TK and Sternberg CN: Pazopanib: clinical development of a potent anti-angiogenic drug. Crit Rev Oncol Hematol 77: 163-171, 2011.

12. Sleijfer S, Ray-Coquard I, Papai Z, et al: Pazopanib, a multikinase angiogenesis inhibitor, in patients with relapsed or refractory advanced soft tissue sarcoma: a phase II study from the European organisation for research and treatment of cancersoft tissue and bone sarcoma group (EORTC study 62043). J Clin Oncol 27: 3126, 2009.

13. Van der Graaf WT, Blay JY, Chawla SP, et al: Pazopanib for metastatic soft-tissue sarcoma (PALETTE): a randomised, double-blind, placebo-controlled phase 3 trial. Lancet 379: 1879-1886, 2012

14. Van Der Graaf W, Blay JY, Chawla SP, et al: PALETTE: Final overall survival (OS) data and predictive factors for OS of EORTC 62072/GSK VEG110727, a randomized, double-blind phase III trial of pazopanib versus placebo in advanced soft tissue sarcoma (STS) patients. J Clin Oncol 30: abs. 10009, 2012. 